\title{
Global output regulation for a class of lower triangular nonlinear systems:
}

DOI:

10.1016/j.automatica.2016.11.008

\section{Document Version}

Accepted author manuscript

Link to publication record in Manchester Research Explorer

\section{Citation for published version (APA):}

Yang, J., \& Ding, Z. (2017). Global output regulation for a class of lower triangular nonlinear systems: A feedback domination approach. Automatica, 76, [15-1454]. https://doi.org/10.1016/j.automatica.2016.11.008

\section{Published in:}

Automatica

\section{Citing this paper}

Please note that where the full-text provided on Manchester Research Explorer is the Author Accepted Manuscript or Proof version this may differ from the final Published version. If citing, it is advised that you check and use the publisher's definitive version.

\section{General rights}

Copyright and moral rights for the publications made accessible in the Research Explorer are retained by the authors and/or other copyright owners and it is a condition of accessing publications that users recognise and abide by the legal requirements associated with these rights.

\section{Takedown policy}

If you believe that this document breaches copyright please refer to the University of Manchester's Takedown Procedures [http://man.ac.uk/04Y6Bo] or contact uml.scholarlycommunications@manchester.ac.uk providing relevant details, so we can investigate your claim.

\section{OPEN ACCESS}




\title{
Global output regulation for a class of lower triangular nonlinear systems: A feedback domination approach *
}

\author{
Jun Yang ${ }^{\mathrm{a}}$, Zhengtao Ding ${ }^{\mathrm{b}}$ \\ a School of Automation, Southeast University, Key Laboratory of Measurement and Control of CSE, Ministry of Education, \\ Nanjing 210096, China \\ ${ }^{\mathrm{b}}$ School of Electrical and Electronic Engineering, University of Manchester, Sackville Street Building, Manchester M13 9PL,
} $U K$

\begin{abstract}
An alternative but new nonlinear output regulation approach is proposed for a class of lower triangular nonlinear systems by using the tool of adding a power integrator. The invariant manifold is solved and represented in terms of various derivatives of disturbances and references. As such, it is unnecessary to suppose that the external disturbances being governed by certain exosystems anymore. The nonlinearities are not restricted to be sufficiently smooth due the the utilization of feedback domination approach. It is shown that the time taken to reach the desired invariant manifold from any initial states under external disturbances is guaranteed to be finite time. The control law is concise in structure for implementation since the feedback domination is implemented in each of the recursive design step.
\end{abstract}

Key words: Lower triangular nonlinear systems; output regulation; finite-time control; feedback domination.

\section{Introduction}

Output regulation is one of the central design problems in nonlinear control theory (Isidori \& Byrnes, 1990; Ding, 2001; Huang \& Chen, 2004; Pavlov, van de Wouw \& Hijmeijer, 2006). It aims to achieve precise reference tracking and disturbance rejection while maintaining system stability (Huang, 2004; Ding, 2013; Chen \& Huang, 2014). The remarkable properties of most output regulation approaches are highlighted as follows: 1) the external disturbances are generally periodic signals supposed to be governed by some neutral stable exosystems (Isidori \& Byrnes, 1990; Pavlov, van de Wouw \& Hijmeijer, 2006; Ding, 2013); 2) the nonlinearities are usually assumed to be sufficiently smooth with respect to their arguments (Chen \& Huang, 2014); and 3) globally/semi-globally/locally asymptotical output regulation is a general control design goal which indicates that the time taken to achieve the target of

* This paper was not presented at any IFAC meeting. This work is supported in part by National Natural Science Foundation of China (Nos. 61573099 and 61633003), Natural Science Foundation of Jiangsu Province (No. BK2012327), and Fundamental Research Funds for the Central Universities (Nos. 2242016R30011 and 2242016K41067).

Email addresses: j.yang84@seu.edu.cn (Jun Yang), zhengtao.ding@manchester.ac.uk (Zhengtao Ding). precise tracking is actually infinite (Isidori \& Byrnes, 1990; Ding, 2013; Chen \& Huang, 2014).

In this communique, we aim to address the output regulation problem in a new perspective for a class of lower triangular nonlinear system subject to general disturbances depicted by (Chen \& Huang, 2014)

$$
\begin{aligned}
\dot{x}_{i} & =x_{i+1}+f_{i}\left(\bar{x}_{i}, w\right), i \in \mathbb{N}_{1: n-1} \\
\dot{x}_{n} & =u+f_{n}\left(\bar{x}_{n}, w\right) \\
y & =x_{1}
\end{aligned}
$$

where $\bar{x}_{i} \triangleq\left[x_{1}, x_{2}, \cdots, x_{i}\right]^{T} \in \mathbb{R}^{i}, u(t) \in \mathbb{R}$ and $y(t) \in \mathbb{R}$ are system state, control input and controlled output, respectively. $w(t) \in \mathbb{D} \subset \mathbb{R}^{p}$ denotes the external disturbance. The regulation error is defined as $\varepsilon(t)=y(t)-$ $y_{r}(t)$ where $y_{r}(t)$ is the desired reference signal.

The development of the new output regulation approach is divided into the following two steps. First, without resorting to any specific exosystems, the desired invariant manifold is solved and depicted by different time derivatives of both reference and disturbance signals. Second, to relaxing the assumption on sufficient smoothness of nonlinearities, the new output regulation law is developed via a feedback domination approach by means of the tool of adding a power integrator (Lin \& Qian, 2000; Polendo \& Qian, 2007). It is further dis- 
cussed that the finite time output regulation is achievable with some mild assumption on the homogeneous degree of the system. Since many useful estimators and differentiators like disturbance observers (Kim, Rew \& Kim, 2010) and higher-order sliding mode differentiators (Levant, 2003) provide adequate means to get the different time derivatives of disturbances, the only assumption on solvability of the new output regulation problem is that the external disturbance shall be measured by sensors. This assumption is well received in certain circumstances among control society community, for example, see full information output regulation (Huang, 2004) and feedforward control (Bequette, 2002) largely in process industries. In a summary, the distinct features of the new output regulation approach are concluded as follows:

1) there is no need to suppose that the disturbances are governed by certain neutral stable exosystems, which relaxes the restrictions on disturbances;

2) the nonlinear items in the system is not required to be sufficiently smooth but only needed to satisfy some homogenous growth condition;

3) the time taken to reach the desired invariant manifold from any initial states is guaranteed to be finite rather than infinite.

\section{Notations and Some Useful Lemmas}

Notations: The symbols $\mathbb{R}_{\text {odd }}^{+}$and $\mathbb{R}_{\text {odd }}^{\geq 1}$ denote the set of ratio of positive odd integers and set of ratio of positive odd integers and greater than 1, respectively. For integers $j$ and $k$ satisfying $0 \leq j \leq k$, let $\mathbb{N}_{j: k} \triangleq\{j, j+1, \cdots, k\}$ be a set of nonnegative integers. The symbol $\mathcal{C}^{i}$ denotes the set of all differentiable functions whose first $i$ th time derivatives are continuous. For $\pi_{i} \in \mathbb{R}, \eta_{i} \in \mathbb{R}, w \in \mathbb{R}^{p}$ and $y_{r} \in \mathbb{R}$, denote $\bar{\pi}_{i} \triangleq\left[\pi_{1}, \pi_{2}, \cdots, \pi_{i}\right]^{T}, \bar{\eta}_{i} \triangleq\left[\eta_{1}, \eta_{2}, \cdots, \eta_{i}\right]^{T}$, $\bar{w}_{i} \triangleq\left\{w, \dot{w} \cdots, w^{[i-2]}\right\}, \bar{y}_{i}^{r} \triangleq\left\{y_{r}, \dot{y}_{r} \cdots, y_{r}^{[i-1]}\right\}$. The symbols $f_{i}^{x}$ and $f_{i}^{\pi}$ are shorts for $f_{i}\left(\bar{x}_{i}, w\right)$ and $f_{i}\left(\bar{\pi}_{i}, w\right)$, respectively. Let $\|w(t)\|_{\infty}=\max _{i \in \mathbb{N}_{1: p}}\left\{\sup _{t \geq 0}\left|w_{i}(t)\right|\right\}$ be the infinity norm of vector $w(t)$. The symbol $\mathcal{L}_{\infty}$ represents the set of all signals whose infinity-norms are bounded. Define a $\mathcal{C}_{0}$ function as $\operatorname{sig}^{\alpha}(\cdot) \triangleq|\cdot|^{\alpha} \operatorname{sgn}(\cdot)$ with $\operatorname{sgn}(\cdot)$ denoting the standard signum function.

The following two lemmas are instrumental for the development of the main results of the paper.

Lemma 1 (Polendo \& Qian, 2007) For $x \in \mathbb{R}, y \in$ $\mathbb{R}, \ell \geq 1$ is a constant, the following inequalities hold $|x+y|^{\ell} \leq 2^{\ell-1}\left|x^{\ell}+y^{\ell}\right|,(|x|+|y|)^{\frac{1}{\ell}} \leq|x|^{\frac{1}{\ell}}+|y|^{\frac{1}{\ell}} \leq$ $2^{\frac{\ell-1}{\ell}}(|x|+|y|)^{\frac{1}{\ell}}$. If $\ell \in \mathbb{R}_{\text {odd }}^{\geq 1}$, then $|x-y|^{\ell} \leq 2^{\ell-1}\left|x^{\ell}-y^{\ell}\right|$ and $\left|x^{\frac{1}{\ell}}-y^{\frac{1}{\ell}}\right| \leq 2^{\frac{\ell-1}{\ell}}|x-y|^{\frac{1}{\ell}}$.

Lemma 2 (Lin \& Qian, 2000) For $x \in \mathbb{R}, y \in \mathbb{R}, c>$ $0, d>0$ and any real-valued function $\gamma(x, y)>0$, the following inequality holds $|x|^{c}|y|^{d} \leq \frac{c}{c+d} \gamma(x, y)|x|^{c+d}+$ $\frac{d}{c+d} \gamma^{-\frac{c}{d}(c+d)}(x, y)|y|^{c+d}$.

\section{Main Results}

\subsection{System Pretreatment}

With some brief derivations, the steady-state of the system is given by $x_{1 s}=\pi_{1}\left(\bar{y}_{1}^{r}\right), x_{i s}=\pi_{i}\left(\bar{w}_{i}, \bar{y}_{i}^{r}\right), u_{s}=$ $\pi_{u}\left(\bar{w}_{n+1}, \bar{y}_{n+1}^{r}\right)$ with $\pi_{i}$ and $\pi_{u}$ determined by the following recursive calculations

$$
\pi_{1}=y_{r}, \pi_{i}=\frac{d \pi_{i-1}}{d t}-f_{i-1}^{\pi}, \pi_{u}=\frac{d \pi_{n}}{d t}-f_{n}^{\pi}
$$

for $i \in \mathbb{N}_{2: n}$. Clearly, the set $\Omega_{x, u} \triangleq\left\{(x, u) \in\left(\bar{\pi}_{n}, \pi_{u}\right)\right\}$ is an invariant manifold for the system (1).

Defining auxiliary variables $\eta_{i}=x_{i}-\pi_{i}\left(i \in \mathbb{N}_{1: n}\right)$, the dynamic system (1) is governed by

$$
\begin{aligned}
\dot{\eta}_{i} & =\eta_{i+1}+f_{i}^{x}-f_{i}^{\pi}, i \in \mathbb{N}_{1: n-1} \\
\dot{\eta}_{n} & =u-\pi_{u}+f_{n}^{x}-f_{n}^{\pi}
\end{aligned}
$$

where $\pi_{i}$ and $\pi_{u}$ (the solutions of output regulation equations (2)) are in terms of the disturbances, the reference signal and their higher-order derivatives, rather than represented by exosystems in Huang \& Chen (2004).

The requirements on disturbances and system nonlinearities are presented and discussed as follows.

Assumption 1 The external disturbances and reference signal satisfy $w(t) \in \mathcal{C}^{n}, y_{r}(t) \in \mathcal{C}^{n}, \frac{d^{i} w(t)}{d t^{i}} \in \mathcal{L}_{\infty}$ and $\frac{d^{i} y_{r}(t)}{d t^{i}} \in \mathcal{L}_{\infty}$ bounded by

$$
\left\|\frac{d^{i} w(t)}{d t^{i}}\right\|_{\infty} \leq \gamma_{w}^{+},\left\|\frac{d^{i} y_{r}(t)}{d t^{i}}\right\|_{\infty} \leq \gamma_{r}^{+}
$$

for $i \in \mathbb{N}_{0: n}$, where $\gamma_{r}^{+}$and $\gamma_{w}^{+}$are some positive constants.

Assumption 2 There exists a constant $\tau$ (a ratio of an even and an odd integer, which is called homogeneous degree) and an $\mathcal{C}^{0}$ function $\gamma_{i}\left(\bar{\eta}_{i}\right)>0$ such that for all $\bar{x}_{i} \in \mathbb{R}^{i}, \bar{\pi}_{i} \in \mathbb{R}^{i}$ and $w \in \mathbb{D} \subset \mathbb{R}^{p}$ with $\mathbb{D}$ being a compact set, the following inequality holds

$$
\left|f_{i}^{x}-f_{i}^{\pi}\right| \leq \gamma_{i}\left(\bar{\eta}_{i}\right) \sum_{j=1}^{i}\left|\eta_{j}\right|^{\frac{r_{i}+\tau}{r_{j}}}, i \in \mathbb{N}_{1: n}
$$

with $r_{1}=1, r_{i}+\tau=r_{i+1} \in \mathbb{R}_{\text {odd }}^{+}$and $\gamma_{i}\left(\bar{\eta}_{i}\right)$.

Remark 1 The disturbances satisfying Assumption 1 represent quite a broad class of exogenous signals. For example, the smooth disturbances governed by neutral stable linear/nonlinear exosystem system (Huang, 2004; Ding, 2013) and general smooth non-periodic disturbances are special cases of this assumption.

Remark 2 Assumption 2 is called homogeneous growth condition (Polendo \& Qian, 2007), which covers a large class of nonlinear functions. Taking any $\mathcal{C}^{1}$ nonlinear function $f_{i}\left(\bar{x}_{i}, w\right)$ as an example, it can be shown that there exists a homogeneous degree $-1<\tau \leq 0$ and a $\mathcal{C}^{0}$ 
function $\gamma_{i}\left(\bar{\eta}_{i}\right)>0$ such that the inequality (5) is satisfied. In addition, for certain $\mathcal{C}^{0}$ but nonsmooth nonlinearities, the inequality (5) is also satisfied by choosing an appropriate homogeneous degree $\tau$. For example, the nonlinearity $f_{1}^{x}=w_{1} x_{1}^{1 / 3}$ satisfies (5) with $\tau=-2 / 3$ and $\gamma_{1}>2^{2 / 3}\left|w_{1}\right|$.

\subsection{New Approach to Output Regulation}

The new approach is developed by using a basic concept of invariant manifold as well as an enhanced version of backstepping approach, namely adding a power integrator (Lin \& Qian, 2000). The detailed derivation of the new output regulation approach consists of three steps described as follows:

Step 1: Let $\sigma, \rho \in \mathbb{R}_{\text {odd }}^{+}$satisfy $\sigma \geq \max _{i \in \mathbb{N}_{1: n}}\left\{r_{i}\right\}, \rho \geq$ $\max _{i \in \mathbb{N}_{1: n}}\left\{r_{i}+\tau, \sigma\right\}$. Design a positive definite and proper Lyapunov function candidate

$$
V_{1}=\int_{0}^{\eta_{1}}\left(s^{\frac{\sigma}{r_{1}}}-\eta_{1}^{* \frac{\sigma}{r_{1}}}\right)^{\frac{2 \rho-r_{2}}{\sigma}} d s
$$

where $\eta_{1}^{*}$ is the first step virtual control law assigned as $\eta_{1}^{*}=0$. By virtue of the inequality (5) in Assumption 2, the time derivative of $V_{1}$ along $(3)$ is

$$
\begin{aligned}
\dot{V}_{1} & =\eta_{1}^{\frac{2 \rho-r_{2}}{r_{1}}}\left(\eta_{2}+f_{1}^{x}-f_{1}^{\pi}\right) \\
& \leq \eta_{1}^{\frac{2 \rho-r_{2}}{r_{1}}}\left(\eta_{2}-\eta_{2}^{*}+\eta_{2}^{*}\right)+\gamma_{1}\left(\eta_{1}\right) \eta_{1}^{\frac{2 \rho}{r_{1}}}
\end{aligned}
$$

The virtual control law $\eta_{2}^{*}$ is designed as

$$
\eta_{2}^{*}=-k_{1}\left(\eta_{1}\right) \xi_{1}^{\frac{r_{2}}{\sigma}}
$$

with $\xi_{1}=\eta_{1}^{\frac{\sigma}{r_{1}}}-\eta_{1}^{* \frac{\sigma}{r_{1}}}$ and $k_{1}\left(\eta_{1}\right)$ is the control gain assigned such that $k_{1}\left(\eta_{1}\right) \geq n+\gamma_{1}\left(\eta_{1}\right)$. Substituting the control law (8) into (7) gives

$$
\dot{V}_{1} \leq \eta_{1}^{\frac{2 \rho-r_{2}}{r_{1}}}\left(\eta_{2}-\eta_{2}^{*}\right)-n \eta_{1}^{\frac{2 \rho}{r_{1}}}
$$

Step $i-1$ : Suppose at step $i-1$, there exists a $\mathcal{C}^{1}$ Lyapunov function candidate $V_{i-1}: \mathbb{R}^{i-1} \rightarrow \mathbb{R}$, which is positive definite and proper, and a set of $\mathcal{C}^{0}$ virtual controllers $\eta_{1}^{*}=0$ and $\eta_{2}^{*}, \cdots, \eta_{i}^{*}$ defined by

$$
\eta_{j}^{*}=-k_{j-1}\left(\bar{\eta}_{j-1}\right) \xi_{j-1}^{\frac{r_{j}}{\sigma}}, \xi_{j}=\eta_{j}^{\frac{\sigma}{r_{j}}}-\eta_{j}^{* \frac{\sigma}{r_{j}}}, j \in \mathbb{N}_{2: i}
$$

with nonlinear control gains $k_{j-1}\left(\bar{\eta}_{j-1}\right)>0$ such that

$$
\dot{V}_{i-1} \leq \xi_{i-1}^{\frac{2 \rho-r_{i}}{\sigma}}\left(\eta_{i}-\eta_{i}^{*}\right)-(n+2-i) \sum_{j=1}^{i-1} \xi_{j}^{\frac{2 \rho}{\sigma}}
$$

It is obvious that (11) reduces to the inequality (9) when $i=2$ under the definitions of (10). In what follows, we will show that inequality (11) also holds at step $i$. To proceed further, a $\mathcal{C}^{1}$ candidate Lyapunov function at step $i$ is defined as

$$
V_{i}=V_{i-1}+W_{i}
$$

where $W_{i}\left(\eta_{1}, \cdots, \eta_{i}\right)=\int_{\eta_{i}^{*}}^{\eta_{i}}\left(s^{\frac{\sigma}{r_{i}}}-\eta_{i}^{* \frac{\sigma}{r_{i}}}\right)^{\frac{2 \rho-r_{i+1}}{\sigma}} d s$ With inequality (11) in mind, the derivative of $V_{i}$ along (3) is given by

$$
\begin{aligned}
\dot{V}_{i}= & \dot{V}_{i-1}+\xi_{i}^{\frac{2 \rho-r_{i+1}}{\sigma}} \dot{\eta}_{i}+\sum_{l=1}^{i-1} \frac{\partial W_{i}}{\partial \eta_{l}} \dot{\eta}_{l} \\
\leq & \xi_{i-1}^{\frac{2 \rho-r_{i}}{\sigma}}\left(\eta_{i}-\eta_{i}^{*}\right)-(n+2-i) \sum_{j=1}^{i-1} \xi_{j}^{\frac{2 \rho}{\sigma}} \\
& +\xi_{i}^{\frac{2 \rho-r_{i+1}}{\sigma}}\left(\eta_{i+1}-\eta_{i+1}^{*}\right)+\sum_{l=1}^{i-1} \frac{\partial W_{i}}{\partial \eta_{l}} \dot{\eta}_{l} \\
& +\xi_{i}^{\frac{2 \rho-r_{i+1}}{\sigma}}\left(\eta_{i+1}^{*}+f_{i}^{x}-f_{i}^{\pi}\right)
\end{aligned}
$$

for a virtual controller $\eta_{i+1}^{*}$ to be determined later. To proceed the inductive proof, the following propositions are required.

Proposition 1 With the help of Lemmas 1 and 2, combining the conditions in Assumptions 1 and 2, we have the following estimations

$$
\begin{aligned}
\left|\xi_{i-1}^{\frac{2 \rho-r_{i}}{\sigma}}\left(\eta_{i}-\eta_{i}^{*}\right)\right| & \leq \frac{1}{3} \xi_{i-1}^{\frac{2 \rho}{\sigma}}+\bar{c}_{i} \xi_{i}^{\frac{2 \rho}{\sigma}} \\
\left|\xi_{i}^{\frac{2 \rho-r_{i+1}}{\sigma}}\left(f_{i}^{x}-f_{i}^{\pi}\right)\right| & \leq \frac{1}{2} \sum_{j=1}^{i-2} \xi_{j}^{\frac{2 \rho}{\sigma}}+\frac{1}{3} \xi_{i-1}^{\frac{2 \rho}{\sigma}}+\hat{c}_{i}\left(\bar{\eta}_{i}\right) \xi_{i}^{\frac{2 \rho}{\sigma}} \\
\sum_{l=1}^{i-1}\left|\frac{\partial W_{i}}{\partial \eta_{l}} \dot{\eta}_{l}\right| & \leq \frac{1}{2} \sum_{j=1}^{i-2} \xi_{j}^{\frac{2 \rho}{\sigma}}+\frac{1}{3} \xi_{i-1}^{\frac{2 \rho}{\sigma}}+\tilde{c}_{i}\left(\bar{\eta}_{i}\right) \xi_{i}^{\frac{2 \rho}{\sigma}}
\end{aligned}
$$

The first inequality in (14) is directly follows from Polendo \& Qian (2007). The proofs of the last two inequalities in (14) are shown in the Appendix. that

With the help of Proposition 1, it follows from (13)

$$
\begin{aligned}
\dot{V}_{i} \leq & \xi_{i}^{\frac{2 \rho-r_{i+1}}{\sigma}}\left(\eta_{i+1}-\eta_{i+1}^{*}\right)-(n+1-i) \sum_{j=1}^{i-1} \xi_{j}^{\frac{2 \rho}{\sigma}} \\
& +\xi_{i}^{\frac{2 \rho-r_{i+1}}{\sigma}} \eta_{i+1}^{*}+c_{i}\left(\bar{\eta}_{i}\right) \xi_{i}^{\frac{2 \rho}{\sigma}}
\end{aligned}
$$

Design a virtual control law as $\eta_{i+1}^{*}=-k_{i}\left(\bar{\eta}_{i}\right) \eta_{i}^{\frac{r_{i+1}}{\sigma}}$ with $k_{i}\left(\bar{\eta}_{i}\right)>n+1-i+c_{i}\left(\bar{\eta}_{i}\right)$. Substituting the virtue control law $\eta_{i+1}^{*}$ into (15) yields

$$
\dot{V}_{i} \leq \xi_{i}^{\frac{2 \rho-r_{i+1}}{\sigma}}\left(\eta_{i+1}-\eta_{i+1}^{*}\right)-(n+1-i) \sum_{j=1}^{i} \xi_{j}^{\frac{2 \rho}{\sigma}}
$$


This completes the inductive proof.

Step n: At the final step, the control is designed as

$$
u=\eta_{i+1}=\eta_{i+1}^{*}=-k_{n}\left(\bar{\eta}_{n}\right) \xi_{n}^{\frac{r_{n+1}}{\sigma}}+\pi_{u}
$$

The above result is summarized by the following theorem.

Theorem 1 Suppose that the disturbances and nonlinearities of system (1) satisfy Assumptions 1 and 2, respectively. The output regulation problem of the system (1) is always solvable by the regulation laws (10) and (17) with the control gains assigned such that $k_{i}\left(\bar{\eta}_{i}\right)>$ $n+1-i+c_{i}\left(\bar{\eta}_{i}\right)$ for $i \in \mathbb{N}_{1: n}$.

Proof: Substituting the regulation law (17) into (16) gives

$$
\dot{V}_{n} \leq-\sum_{j=1}^{n} \xi_{j}^{\frac{2 \rho}{\sigma}}
$$

Using LaSalle invariant principal, it follows from (18) that $\xi_{i}(t) \rightarrow 0, \eta_{i}(t) \rightarrow 0, \varepsilon(t) \rightarrow 0$ and subsequently $x_{i}(t) \rightarrow \pi_{i}(t)$ as $t \rightarrow \infty$.

The control gain $k_{i}\left(\bar{\eta}_{i}\right)$ plays an important role in shaping the control performance of the closed-loop system. The larger the control gain, the faster the convergence rate but the higher the control energy and vice versa. In addition to the above globally asymptotically output regulation, we further present the result of finitetime output regulation problem as follows.

Theorem 2 Suppose that the disturbances and nonlinearities of system (1) satisfy Assumptions 1 and 2, respectively. If the homogenous degree $\tau$ satisfies $-1<\tau<0$, the finite-time output regulation problem of the system (1) is solvable by the regulation laws (10) and (17).

Proof: By Lemma 1, it can be verified that the following holds

$$
\begin{aligned}
V_{n} & =\sum_{j=1}^{n} \int_{\eta_{j}^{*}}^{\eta_{j}}\left(s^{\frac{\sigma}{r_{j}}}-\eta_{j}^{* \frac{\sigma}{r_{j}}}\right)^{\frac{2 \rho-r_{j+1}}{\sigma}} d s \\
& \leq \sum_{j=1}^{n}\left|\eta_{j}-\eta_{j}^{*}\right| \cdot\left|\xi_{j}\right|^{\frac{2 \rho-r_{j+1}}{\sigma}} \leq \mu \sum_{j=1}^{n}\left|\xi_{j}\right|^{\frac{2 \rho-\tau}{\sigma}}
\end{aligned}
$$

where $\mu$ is a positive constant. Let $\lambda=\mu^{(2 \rho-\tau) / 2 \rho} / 2$. Using Lemma 1, it concludes from (18) and (19) that

$$
\dot{V}_{n}+\lambda V_{n}^{\frac{2 \rho}{2 \rho-\tau}} \leq-\frac{1}{2} \sum_{j=1}^{n}\left|\xi_{j}\right|^{\frac{2 \rho}{\sigma}}
$$

Using Theorem 2.2 in Huang, Lin \& Yang (2005), it follows from $(20)$ that $\xi_{i}(t) \rightarrow 0$ and $\eta_{i}(t) \rightarrow 0$ for $i \in \mathbb{N}_{1: n}$ in finite time, which implies that $\varepsilon(t) \rightarrow 0$ and $x_{i}(t) \rightarrow \pi_{i}(t)$ in finite time.
Remark 3 The disturbances in many practical control systems are measurable by sensors (Bequette, 2002). However, the derivatives of disturbances are not always measurable. Consequently, we introduce a higher-order sliding mode differentiator (Levant, 2003; Shtessel, Shkolnikov, \& Levant, 2007) to gain the derivatives of disturbances

$$
\begin{aligned}
\dot{z}_{0} & =v_{0}=-l_{0} \operatorname{sig}^{\frac{n-1}{n}}\left(z_{0}-w(t)\right)+z_{1}, \\
\dot{z}_{i} & =v_{i}=-l_{i} \operatorname{sig}^{\frac{n-1-i}{n-i}}\left(z_{i}-v_{i-1}\right)+z_{i+1}, \\
\dot{z}_{n-1} & =-l_{n-1} \operatorname{sig}^{0}\left(z_{n-1}-v_{n-2}\right),
\end{aligned}
$$

where $l_{i}=\lambda_{i} L^{1 /(n-i)}$ with $\lambda_{i}>0$ and $L>\gamma_{w}^{+}$are observer coefficients to be designed. The error dynamics of the differentiator (21) is governed by

$$
\begin{aligned}
\dot{e}_{0} & =-l_{0} \operatorname{sig}^{\frac{n-1}{n}}\left(e_{0}\right)+e_{1} \\
\dot{e}_{i} & =-l_{i} \operatorname{sig}^{\frac{n-i-1}{n-i}}\left(e_{i}-\dot{e}_{i-1}^{i}\right)+e_{i+1} \\
\dot{e}_{n-1} & \in-l_{n-1} \operatorname{sig}^{0}\left(e_{n-1}-\dot{e}_{n-2}\right)+\left[-\gamma_{w}^{+}, \gamma_{w}^{+}\right]
\end{aligned}
$$

where $e_{i}(t)=z_{i}(t)-d^{i} w(t) / d t^{i}$ denotes the differentiator errors. The error dynamic system (22) will converge to zero after a finite time transient process, which implies that $w^{[i]}(t) \equiv z^{i}(t)$ for all $t \geq T$ (Levant, 2003). This paper mainly focuses on developing a new nonlinear output regulation approach for a class of lowertriangular nonlinear systems. It is actually a version of full information approach. Apart from the differentiator above, all existing disturbance observers can be applied for the presented approach as long as they can obtain the disturbance information timely and accurately.

\section{An Illustrative Example}

Consider the following nonlinear dynamic system under disturbances

$$
\begin{aligned}
& \dot{x}_{1}=x_{2}+0.5\left[\sin \left(w_{1}(t)\right)+w_{2}(t)\right] x_{1}^{\frac{3}{5}}+w_{2}(t) \\
& \dot{x}_{2}=10 u+\left[w_{1}^{2}(t)+w_{2}(t)\right] x_{2} x_{1}^{2}+w_{1}(t) w_{2}(t)
\end{aligned}
$$

The reference signal to be tracked is given by $y_{r}(t)=$ $4+\sin (2 t)+2 \cos (t)$. The disturbance signals for the simulation studies are depicted by $w_{1}(t)=-1+\sin (\pi t / 4)+$ $10 t^{2} e^{-2 t}$ and $w_{2}(t)=1.5+\cos (\pi t / 2)$. Let $\tau=-2 / 5$, $r_{1}=1, r_{2}=3 / 5, r_{3}=1 / 5$ and $\sigma=\rho=1$. Some brief calculations reveal that $f_{1}\left(x_{1}, w\right)$ and $f_{2}\left(\bar{x}_{2}, w\right)$ satisfy the condition given in Assumption 2 with $\gamma_{1}\left(\eta_{1}\right)$ a positive constant and $\gamma_{2}\left(\bar{\eta}_{2}\right)=c\left(\eta_{1}^{14 / 5}+\eta_{2}^{14 / 5}+1\right)$ a continuous function.

With the design procedure described in the last section, the finite-time output regulation law for system (23) is given by

$$
\left.u=-k_{2}\left(\bar{\eta}_{2}\right)\right)\left(\eta_{2}^{\frac{5}{3}}+k_{1}^{\frac{5}{3}} \eta_{1}\right)^{\frac{1}{5}}+\pi_{u}
$$



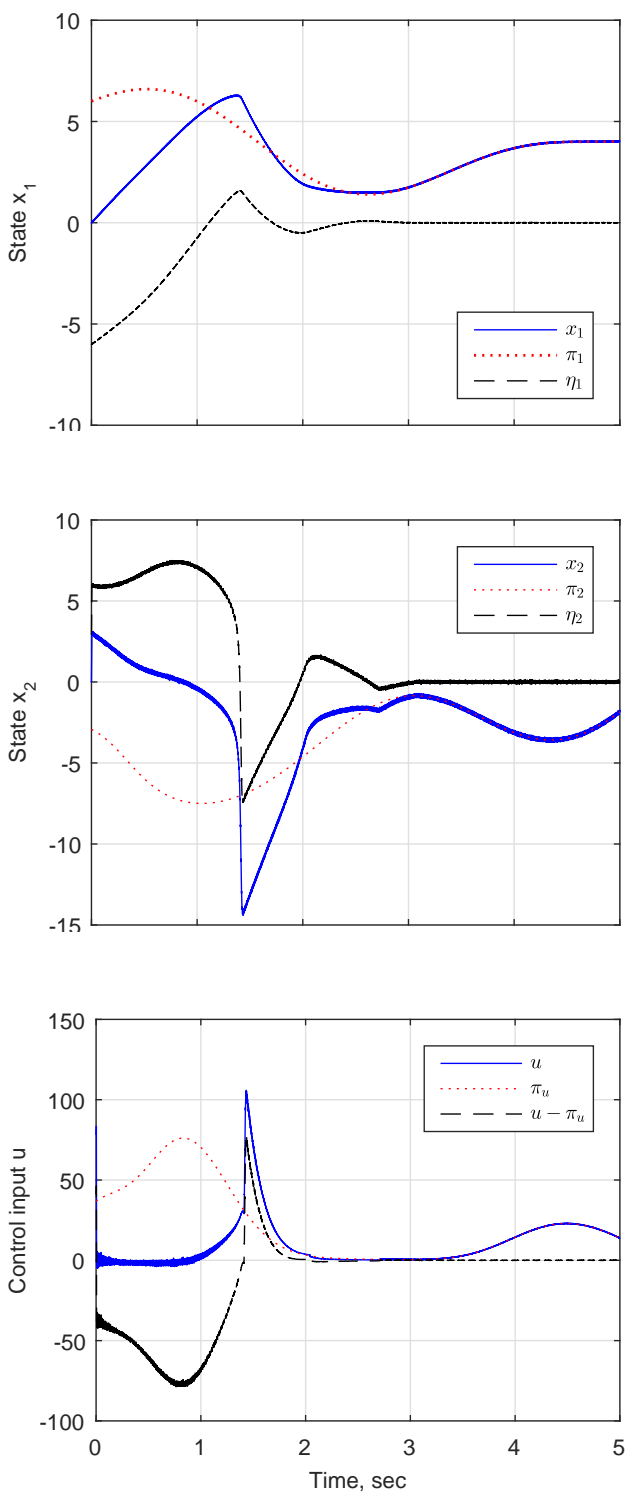

Fig. 1. Response curves of system (23) under finite-time output regulation law (24).

with $k_{2}\left(\bar{\eta}_{2}\right)=k_{2 c}+k_{2 \gamma}\left(\left|\eta_{1}\right|^{3}+\left|\eta_{2}\right|^{3}\right)$. The control parameters are chosen as $k_{1}=2, k_{2 c}=5$ and $k_{2 \gamma}=1$. In addition, a second-order sliding mode differentiator as stated in Remark 3 is utilized to access the derivatives of disturbances, whose parameters are selected as $\lambda_{0}=1.1, \lambda_{1}=1.5$ and $L=5$. The simulations are carried out in a Gaussian measurement noise environment for practicality. Response curves of the states and the control input for system (23) are shown by Fig. 1.

\section{Conclusions}

The global output regulation problem of a class of lower triangular nonlinear system has been reinvestigated by using the tool of adding a power integrator. Thanks to the feedback domination design technique, the newly proposed output regulation law has largely relaxed the restrictions on both the plant and the disturbances. Furthermore, it has been proven that the finite time output regulation is accessible by relaxing the restriction on the homogeneous degree of the nonlinearities of the system. In a conclusion, the proposed method in the communique has provided a complementary methodology to existing nonlinear output regulation theories by using several different tools from a new standpoint.

\section{Appendix: Proof of Proposition 1}

6.1 Proof of the Second Inequality in (14)

Using Lemma 1, the inequality (5) is rewritten as

$$
\begin{aligned}
\left|f_{i}^{x}-f_{i}^{\pi}\right| & \leq \gamma_{i}\left(\bar{\eta}_{i}\right) \sum_{j=1}^{i}\left|\eta_{j}\right|^{\frac{r_{i+1}}{r_{j}}} \\
& =\gamma_{i}\left(\bar{\eta}_{i}\right) \sum_{j=1}^{i}\left|\xi_{j}-k_{j-1}^{\frac{\sigma}{r_{j}}}\left(\bar{\eta}_{j-1}\right) \xi_{j-1}\right|^{\frac{r_{i+1}}{\sigma}} \\
& \leq \bar{\gamma}_{i}\left(\bar{\eta}_{i}\right) \sum_{j=1}^{i}\left|\xi_{j}\right|^{\frac{r_{i+1}}{\sigma}}
\end{aligned}
$$

for some $\mathcal{C}^{0}$ function $\bar{\gamma}_{i}\left(\bar{\eta}_{i}\right)$. Continuing using Lemmas 1 and 2 , it follows from (25) that

$$
\begin{aligned}
& \left|\xi_{i}^{\frac{2 \rho-r_{i+1}}{\sigma}}\left(f_{i}^{x}-f_{i}^{\pi}\right)\right| \\
& \leq \bar{\gamma}_{i}\left(\bar{\eta}_{i}\right)\left|\xi_{i}\right|^{\frac{2 \rho-r_{i+1}}{\sigma}} \sum_{j=1}^{i}\left|\xi_{j}\right|^{\frac{r_{i+1}}{\sigma}} \\
& \quad \leq \frac{1}{2} \sum_{j=1}^{i-2} \xi_{j}^{\frac{2 \rho}{\sigma}}+\frac{1}{3} \xi_{i-1}^{\frac{2 \rho}{\sigma}}+\hat{c}_{i}\left(\bar{\eta}_{i}\right) \xi_{i}^{\frac{2 \rho}{\sigma}}
\end{aligned}
$$

\subsection{Proof of the Last Inequality in (14)}

With Lemmas 1 and 2 in mind, it is derived from (25) that

$$
\begin{aligned}
\left|\dot{\eta}_{l}\right| & =\left|\eta_{l+1}+f_{l}^{x}-f_{l}^{\pi}\right| \\
& \leq\left|\xi_{l+1}\right|^{\frac{r_{l+1}}{\sigma}}+k_{l}\left(\bar{\eta}_{l}\right)\left|\xi_{l}\right|^{\frac{r_{l+1}}{\sigma}}+\bar{\gamma}_{l}\left(\bar{\eta}_{l}\right) \sum_{j=1}^{l}\left|\xi_{j}\right|^{\frac{r_{l+1}}{\sigma}} \\
& \leq \tilde{\gamma}_{l}\left(\bar{\eta}_{l}\right) \sum_{j=1}^{l+1}\left|\xi_{j}\right|^{\frac{r_{l+1}}{\sigma}}
\end{aligned}
$$

With similar derivations with Polendo \& Qian (2007), we can obtain the last inequality of (14).

\section{References}

Bequette, B.W. (2002). Process Control: Modeling, Design and Simulation. Upper Saddle River, NJ: Prentice-Hall. 
Chen, Z., \& Huang, J. (2014). Stabilization and Regulation of Nonlinear Systems. London: Springer.

Ding, Z. (2001). Global output regulation of uncertain nonlinear systems with exogenous signals. Automatica, 37(1), 113-119.

Ding, Z. (2013). Nonlinear and Adaptive Control Systems. London: IET Press.

Huang, J. (2004). Nonlinear Output Regulation: Theory and Applications. Philadelphia: SIAM Press.

Huang, J., \& Chen, Z. (2004). A general framework for tackling the output regulation problem. IEEE Trans. on Auto. Control, 49(12), 2203-2218.

Huang, X., Lin, W., \& Yang, B. (2005). Global finitetime stabilization of a class of uncertain nonlinear systems. Automatica, 41(5), 881-888.

Isidori, A., \& Byrnes, C. (1990). Output regulation of nonlinear systems. IEEE Trans. on Auto. Control, 35(2), 131-140.

Kim, K.S., Rew, K.H., \& Kim, S. (2010). Disturbance observer for estimating higher order disturbances in time series expansion. IEEE Trans. Autom. Control, $55(8), 1905-1911$.

Levant, A. (2003). Higher-order sliding modes, differentiation and output-feedback control. Int. J. Control, 76(9-10), 924-941.

Lin, W., \& Qian, C. (2000). Adding one power integrator: a tool for global stabilization of high-order lowertriangular systems. Syst. Control Lett., 39(5), 339-351.

Pavlov, A., van de Wouw, N., \& Hijmeijer, H. (2006). Uniform Output Regulation of Nonlinear Systems: A Convergent Dynamics Approach. Berlin: Birkhauser.

Polendo, J., \& Qian, C. (2007). A generalized homogeneous domination approach for global stabilization of inhearently nonlinear systems via output feedback. Int. J. Robust Nonlinear Control, 17(7), 605-629.

Shtessel, Y. B., Shkolnikov I. A., \& Levant, A. (2007). Smooth second-order sliding modes: Missile guidance application. Automatica, 43(8), 1470-1476. 